\section{Sense of Place and Design}

RAchelle Lopez

Planning and design has always been about creating and maintaing the quality of the built environment and making it meaningful to its users. Rachelle Lopez discusses the importance of understanding the underlying qualities that convey a sense and a meaning to a place, their relationship to the users' experiences, and their importance for a successful design process.

Understanding the connections between communities and their places is fundamental for planning and design to be meaningful and reflect the true values of communities we work for. Three concepts help us in this endeavor: sense of place, experience of place, and design as place making. Studying these concepts can help us figure out which elements need to be considered when designing in order to maintain or enhance the community feelings for a place or to increase the users' desire to inhabit or experience that place.

Findings of such studies will help us understand the underlying reasons why different places are highly utilized while other places are used at a lower frequency or not at all. It will also guide visioning and design development in a direction that maintains the sense of place of an area, enhances users' experiences and connections, and utilizes design to improve the users' assessments of the place. Overall, this understanding of place will help prevent cookie-cutter designs from being implemented while facilitating planners and designers in creating and maintaining unique environments.

\section{Sense of Place}

According to Lynch (1981: 131), "Sense is the interaction between person and place...[and]...depends on spatial form and quality, culture, temperament, status, experience and current purpose of the observer". A sense of place can be "created by the pattern of reactions that a setting stimulates for a person" (Steele, 1981: 12). These reactions are a product of both the setting's attributes and personal aspects that are brought in by users. The specific experience a person has in a particular setting, and how that setting causes him or her to feel, is what directs and molds an individual's sense of place. Identity is the simplest form of sense and is the extent to which a person can recognize or recall a place as being distinct from other places; in other words, the place is unique and maintains a character of its own that makes it stand apart from other areas.

According to Norberg-Shulz (1979: 5) "spaces where life occurs are places" and are the result of relationships between actions, conceptions, and physical attributes. While an animal's awareness of space is related to survival in terms of food and shelter, a person's awareness of space includes where he or she has grown and spent their time (Trowbridge, 1913: 889). Creating a sense of place can give people the feeling of being at home or having a home that they can go back to while also providing a sense of control over their own fate.

In some instances, people merely see themselves as observers of their environment. At the same time, people also look for and "perceive themselves as being connected to or being a part of their surroundings" (Hiss, 1990: 22). Place then becomes a materialization of peoples' dwellings, where they can identify themselves with the environment and where the environment has meaning to them. People have and continue to identify themselves with places, such as calling them home, and use them to provide a kind of symbolic identity that distinguishes them from others.

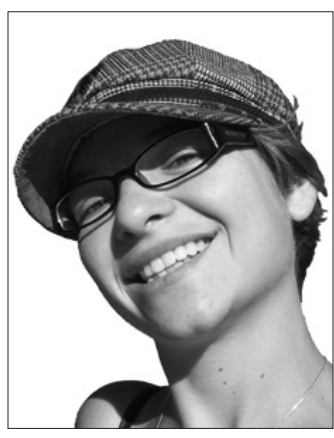

Rachelle Lopez holds a Bachelor of Landscape Architecture and received her Masters in City and Regional Planning from Cal Poly San Luis Obispo in 2009.
Note: This paper is based in the author's thesis Enhancement Plan for Downtown Cayucos, CA presented for Cal Poly's Master of City and Regional Planning, 2009. The thesis can be retrieved from $\mathrm{Cal}$ Poly's Kennedy Library at: <http://digitalcommons. calpoly.edu/theses/171/> 
Canter suggests that "what a place 'is' cannot be fully recognized until we know what behavior is associated with it, what the physical parameters of the setting is, and what the description, or conception, which people have of that physical environment is" (Canter, 1977: 159-160). Observing the differences between people's behavior and their conceptualizations of place make it possible to understand the multiple 'sub-places' that coexist in any given place. Recognizing that places contain more than one use and accommodate many behaviors allows us to understand and provide for the diversity of people that can be accommodated in any given place.

We can begin to see place as "both an object of people's interest, concern, influence, attention, alteration, and enjoyment and the cause of people's feelings, moods, responses, constraints, achievements, survival, and pleasure" (Steele, 1981: 9). Whether seen as object or cause, people will create their own sense of place through different aspects they bring to the setting and how they use the space. Place can also be broken down in terms of space and character. "Space denotes the three-dimensional organization of the elements which make up a place, and character denotes the general atmosphere which is the most comprehensive property of any place" (Norberg-Shulz, 1979: 11). Space is experienced as the three-dimensional extension of the world around us. It changes according to the relationships that are formed between people and their surroundings. In this sense, it can be argued that the formation of this relationship is more dependent on the spatial organization of the designed environment than the use of materials in the area that provide for the place's character.

The various aspects of physical attributes and other elements that create a space also assist in the recognition of places. Based on which attributes are present in a given area, certain groups of people will be able to identify themselves with the space. In order for a place to be successful it "needs to be accessible to all of the senses, engaging the perceptions of its occupants because it is this perception that allows for a direct sense of continuing membership in communities and regions" (Hiss, 1990: xiii). Another factor that aids in a sense of belonging is if the area is in some way either "appropriate to the person and their culture, makes them aware of their community, its past, the web of life, as well as of time and space in which those are contained" (Lynch, 1981: 142).

Figure 1

Downtown Cayucos,

Calif. The area object

of the author's study on sense of place.

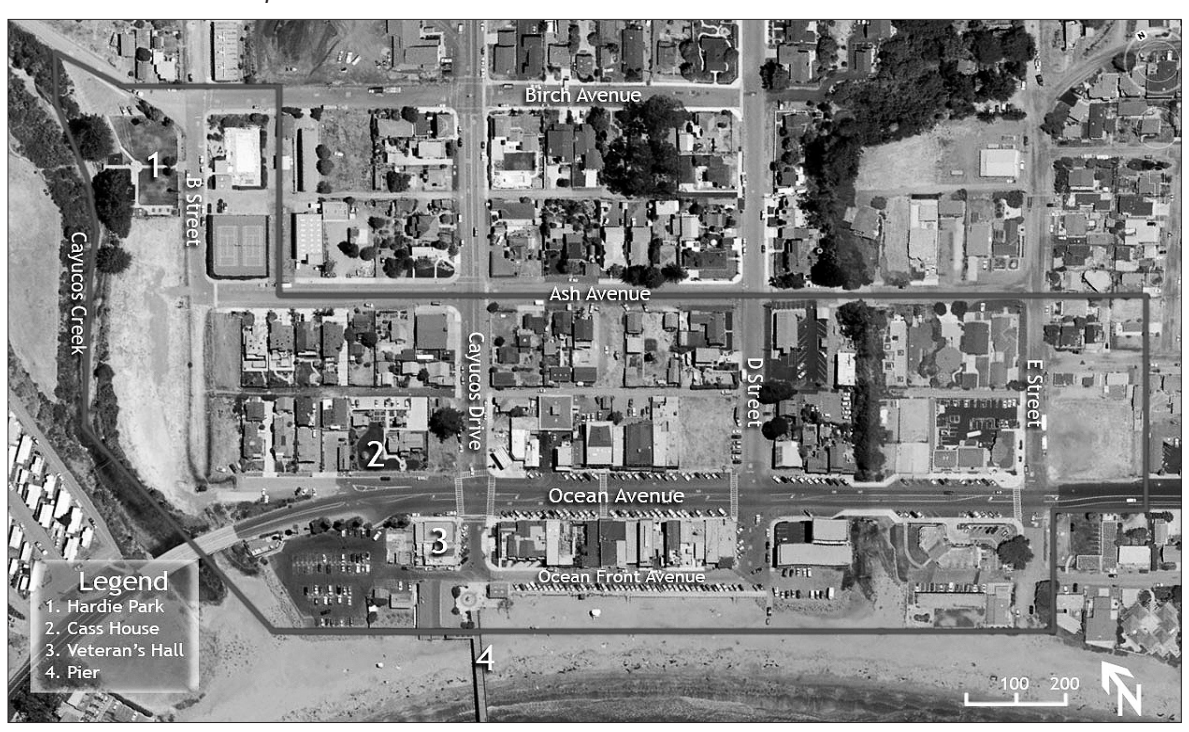

Given the opportunity, it is argued that people and animals will strive to select the habitat that best matches their needs, preferences and lifestyle (Rapoport, 1977: 81). This selectivity is due to motivation, experience and adaptation levels, as well as cognitive needs, such as connectedness, identity, scale, and orientation. It is therefore important for people to understand that they are "an integral part of the environment, and if they forget that, it can only lead to human alienation and environmental disruption" (Norberg-Shulz, 1979: 23). For not only do we observe our environment and have individual and unique perceptions of that environment, but we ourselves are a part of it, participating with other people in creating a sense of place.

\section{Experience of Place}

A person's experience of a place is not believed to revolve only around the function of the area's physical attributes. What could also be taken into account is what 
characteristics are picked up by people's five senses, their history, and their moods while they are experiencing it. Greenbie (1981: ix) suggests that "what each of us actually experiences is selected, shaped and colored by what we know, that is, by what we have already experienced". People's reactions, identifications or perceptions of a place will depend on the type of exposure they had to various kinds of settings throughout their lives. Personal differences that may be displayed by different individuals stem from these differences that exist between people, their concept of place, and the interactions they have had and continue to have with their surroundings.

People's perception of a place also varies depending on their purpose or goal for being there as well as their expectations. Their goal or purpose is what links them to the place, helping form a bond between them and the area. Their expectations link them to a place because of what they intend to find there, for instance, people with similar interests or certain accommodations. As a person continues to experience a place over time, this bond becomes stronger. Canter looks at this relationship between person and place as the person's 'environmental role' suggesting that a person's role is what determines the frequency at which that person relates various places to themselves (Canter, 1977: 178).

People, according to Steele (1981: 4), "experience a sense of place, given the right conditions". This means that a space needs to accommodate for a wide range of individuals in order to ensure their ability to experience a connection with the space. Spaces need to provide for both the 'doers' who actively use the space and the 'watchers' who use the space passively. The fact that people act and behave differently in different settings suggests that people make their behavior fit with and be appropriate to the setting. This implies that the "environment provides cues for behavior through a form of non-verbal communication" (Rapoport, 1977: 3). The area should therefore contain both physical and social attributes to guide behavior and emotional responses of the various users. If these

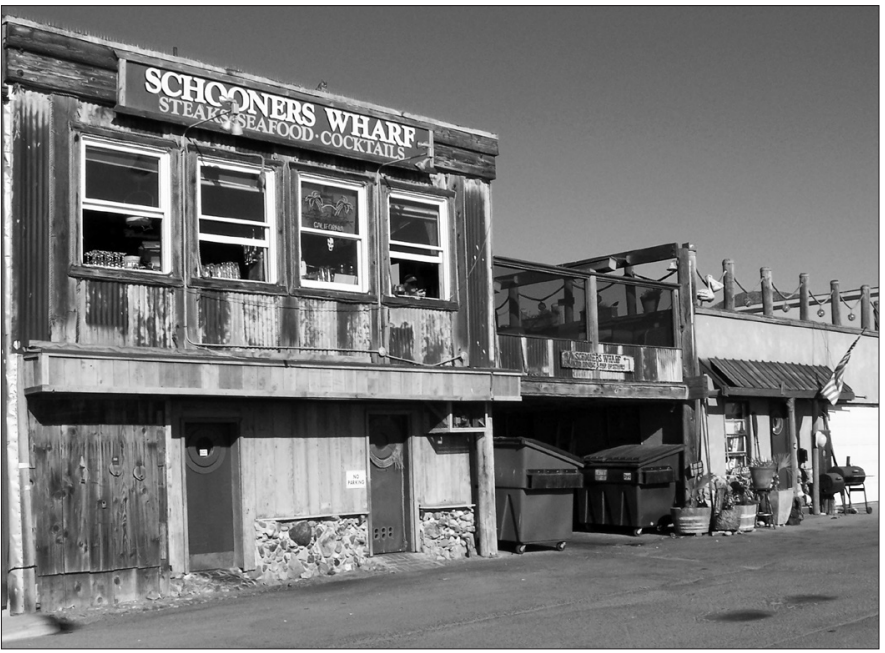

Figure 2

One of the traditional restaurants facing the beach at downtown Cayucos.

Visual quality can affect a person's experience greatly because people respond to what appears before them, visual cues in the area, and what they recall of places. Tuan (1974: 6). believes that "of the traditional five senses man is more consciously dependent on sight to make his way in the world than on the other senses". This allows us to understand and see the space in terms of meaning, appearance, and function. Nasar (1998: 3) furthers this idea stating, "Appearance and meaning are not separate from function but central to it". The disagreements people have about the appearance of a place go beyond what they see to include the experience and the level of satisfaction they get out of a place. For instance, when a place is compatible, say it has a wide sidewalk, people may feel comfortable in it and able to perform tasks, such as walking down the street. When a place feels disorderly, containing graffiti, litter, or is under maintained, it can cause them to feel a sense of anxiety or fear. Therefore, the way in which people may perceive the environment can impact their enjoyment, behavior, and feelings towards a place, which in turn, can contribute to their sense of place.

"The appearance of cities, towns, neighborhoods... affect daily activities by influencing our emotional reactions or affective responses. The aesthetics of an environment can evoke feelings such as pleasure, relaxation, excitement and fear" (Antaov, 1998: 239). These emotional responses in turn can affect behavioral responses, with people taking favorable or unfavorable actions in accordance to how they feel. People tend to respond to 
what they see in relation to a physical form. With a range of visual qualities, attributes, and architecture, users can be attracted to and interested in a designed space for different reasons. Building density, scale, and type are key factors to the design of a space because people are able to create a relationship between their evaluative responses and the building's visual attributes. There has been research done on aesthetic controls showing that the environment plays a significant role in the well-being of people. On the negative side, these controls can create a "recipe for homogeneity, sensory deprivation, and lack of environmental or social identity" (George, 2000: 166). On the positive side, these controls can help coordinate the overall spatial design, evoking a sense of identity and belonging.

Using "a pattern or repetition of specific experiences facilitates a person's ability to increase their sense of place more than if only a single experience was presented in the area" Steele, 1981: 141). Time patterns also influence the experience of a place through time-related elements such as when places are open, how long and when they

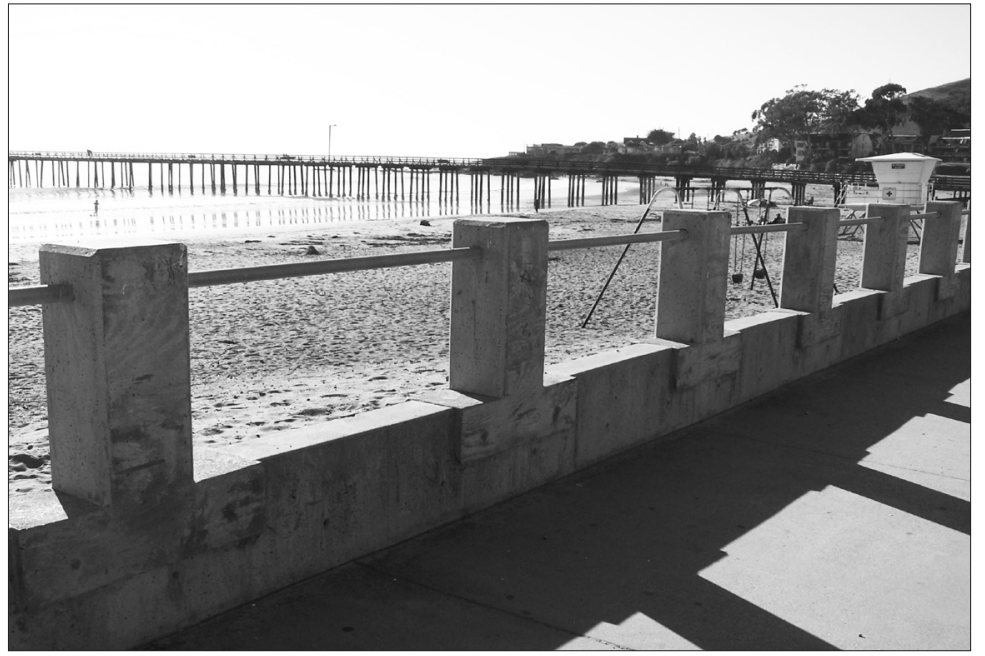

Figure 4

The beach and pier; strong elements for the sense of place in downtown Cayucos. can be used, which services are available at different times, the hours established for work, etc. This and "personal combinations of perceptions, expectations, assumptions, habits, social concerns, and personal fears can prevent people from using everything a setting provides" (Steele, 1981: 173). By having choice and variety in an area, accessibility can be increased and an assortment of needs can be met.

Tuan believes that "open space signifies freedom and the public realm while an enclosed space signifies a cozy, secure and private place" (Tuan, 1974: 27-28). When it comes to open space, there are two suggested requirements that can be met in order to enhance the experience of a person in such a space. First, a person's sense of security in a public space needs to be "spatially anchored" (Hiss, 1990: 87-88). Each user has to be able to find some space within that place, like a niche, where he or she can be without being bothered by other people and without getting in anyone's way. This makes it important for a space to have places of enclosure, such as benches surrounded by vegetation placed to the side of a pathway. This also makes it important for a space to have designated edges. Providing known edges lets people know where one space ends and another begins, such as through pavement patterns to designate walkways from seating areas. Second, people need reasons for going to a place, and the more reasons they have, the more time they'll spend there, the more frequently they will visit and the longer they will stay.

Places, both built and natural, have an effect on how we feel and act, impacting our sense of self, sense of safety and the ways we interact with other people. This means that the "places where we spend our time affect the people we are and can become" (Hiss, 1990: xi). If we are unaware and unable to check on the experiences around us, we will be incapable of recognizing their deterioration or their need for renewal. By paying attention to our experiences, we can strive to maintain places that provide for people's needs, stimulate their senses, and sustain rich experiences.

\section{Design as Place Making}

Design is "to devise for a specific function or end" (Merriam-Webster Dictionary, 1997). Community design is the "art of making sustainable living places that both thrive and adapt to people's needs for shelter, livelihood, 
commerce, recreation, and social order...and can be interpreted as a sense of belonging and way of life" (Hall \& Porterfield, 2001: 3). One of the goals of community design has been for designers to consider that all places are a composition of social, biological, and physical aspects. In addition, design decisions that are made should be looked at in the future tense. Some questions that should be addressed include "What will happen? What is better? Better for whom? And how can better design be achieved and improved?" (Rapoport, 1990: 81). In order to answer these questions, design must be based on knowledge and that knowledge should be concerned with environment-behavior interaction. Design means to "visualize the genius loci, and the task of the designer is to create meaningful places, whereby he helps man to dwell" (Norberg-Shulz, 1979: 5). The design should then make human needs and expectations and environmental features congruent with one another.

"Urban design... is commonly understood with respect to context; it has to do with configuring and constructing a homogenous, coordinated, continuous environment that presents itself with the coherence of a landscape" (Rossi, 1982: 116). Within this cohesive landscape are a series of places different groups of people can relate to. This series of places include things such as districts, edges, corridors, and nodes, common elements studied by Kevin Lynch that help with an area's legibility. Legibility, also referred to as coherence, is important and attributes to the creation of a walkable environment that is enjoyable to pedestrians (Lynch, 1960; Cullen, 1968; Bentley, 1985; Kopec, 2006). It relates to "the way objects in a scene come together to form some sort of context" (Kopec, 2006: 31). This means that an area needs to be recognizable to increase its use.

Besides legibility, according to Lynch (1960), there are two other qualities to be considered in the successful design of a place. These include complexity and mystery. Complexity addresses the number and variety of elements that can be found within a space. By having a variety of elements, the area is able to relate to a wider variety of people and maintain their interest. Finally, mystery is "the degree to which a scene contains hidden information" (Kopec, 2006: 31). This stimulates users to continue moving through the space and explore the area.

There is a fifth component that also needs to be addressed which is time (Lynch, 1976). For a place to maintain user interest it needs to be able to be related to over time. Through the use of these elements and by changing, upgrading, and maintaining the area, user interest is more likely to remain by continuing to arouse users with new elements each time they visit.

In today's society, the prominent pattern in development is the monotonous building replacing the rich diversity of places, leaving no variety, surprise, or traces of the area's own history and development (Steele, 1981: 8). In order to ensure the desired and sought after experiences in an area are still available, we need to make sure that primary components remain in their original positions, preventing the place from becoming unrecognizable. People tend to create and relate strong mental images to sense of place. If a physical form already exists, for instance a historic building, the new design should utilize and reuse it, incorporating these new features with the existing and historic ones, maintaining the visual integrity of the space in regards to a user's mental image. However, flexibility can also be built into an area to allow for any modifications that may be needed in the future. This will increase the durability and duration of a design while preventing the need for reconstruction because user needs could not be met.

What is found is that people act according to their reading of the environmental cues that surround them. The design of the environment might be understood as "a process of encoding information, then the users can be seen as decoding it" and "if the code is not shared, understood or inappropriate, the environment does not communicate" (Rapoport, 1977: 3). Spaces that are long, narrow, and have high complexity levels tend to encourage more dynamic activities, including walking, shopping, and recreation, because there is a built-in sense of enticement 
created by aspects such as hidden views and the lack of accommodation to rest. Rest spaces tend to be more static and wider, frequently containing vegetation and providing sitting accommodations. These areas provide users with places to eat, sit, and watch others. Other things that can affect walking include safety, the ambient environment, distances to amenities, topography, culture, interest, complexity, and physical support structures (such as sidewalks).

According to Mehta (2007: 165), "streets are an important part of public open space...[and]...people depend on streets for functional, social and leisure activities". Streets need to be incorporated into the designed space to further support public life. Areas that have been designed with the street have been seen as economically successful, physically healthy, and having a greater sense of community. By providing a variety of services on the site, a range of people's needs are met and the space is continuously used throughout the day.

People can create what is known as an evaluative image, which "arises from the person and the environment and the ongoing interaction between the two... and may also arise from the content meaning of the form" (Nasar, 1998: 4). Though people have their own unique experiences with their environment, and outsiders tend to evaluate places differently than insiders who are familiar with the area, it has been found that people with similar cultures or socioeconomic backgrounds share common meanings in their evaluative images.

Lund suggests that the social aspect of the environment influences people's perception of their sense of community. This sense of community promotes "social cohesion by aiding the movement of information and ideas within the community and increasing access to resources and opportunities" (Lund, 2002: 302). A variety of spaces, including intimate, personal, social, and public, are needed within the overall designed space in order to accommodate and provide areas for the different levels of interaction that people need sociologically. This includes vegetation, seating, sidewalk and street width, goods and services, personalized storefronts, and permeability between the storefronts and the street.

One can conclude that providing some key features along with a meaningful combination of design elements, a designer can help both visitors and community members to interact more or less intensely with the built environment. Creating these different levels of interaction enhances the users' connection to a specific area, thus recognizing it as a specific 'place' with a specific character, which, in their minds, is distinct from other places.

\section{Conclusion}

All three concepts that were explored, sense of place, experience of place and design influence, can play an important role in guiding design and development goals for the enhancement of an area. In order to maintain an area's sense of place, it needs to remain a place that people recognize as being unique and distinct from other places. The area needs to be legible and be comprised of components that will enable users to make a connection to the environment. Designers and planners can help ensure the success of a place by taking these concepts into consideration; by learning about and understanding a place, they can create better designs that are fitting for that place. Doing so will help each place provide users with the opportunity to connect with their surroundings, accommodate a variety of user needs, stimulate their senses, sustain and enrich user experiences, and provide features that allow for different levels of interaction between all user groups.

Without taking these issues into consideration, places risk losing the ability for users to connect with and create meaningful experiences. This is not to say that nothing new should be added to a place. Rather, it implies finding a balance between the new and the old. This enables the place to maintain its character while still providing it with the ability to progress. Taking these findings into consideration will enable an area to maintain 
a distinct sense of place and enhance the connection it has with its users by guiding design and development in a more suitable direction.

It is important to encourage research into what connects people to places, and to use their findings on a projectby-project basis. For planners and designers, it is fundamental to learn about and understand the place for guidance towards what the community needs and not just what they want to design. This will deter places from being completely redeveloped in a cookie-cutter manner and will generate plans and design solutions that are meaningful to the community and fitting for the area.

\section{References}

Antaov, Anli. 1998. Environmental aesthetics. Journal of Planning Literature 13(2), 239-257.

Bentley, Ian et al. 1985. Responsive Environments. London: Architectural Press.

Canter, David. 1977. The Psychology of Place. London: Architectural Press.

Cullen, Gordon. 1968. Townscape. New York: Reinhold Book Corporation.

Greenbie, Barrie. 1981. Spaces: Dimensions of the Human Landscape. New Haven: Yale University Press.

George, R. Varkki and Marcia Caton Campbell. 2000. Balancing different interests in aesthetic controls. Journal of Planning Education and Research (20), 163-175.

Hall, Kenneth B. and Gerald A. Porterfield. 2001. Community by Design. New York: McGraw-Hill.

Hiss, Tony. 1990. The Experience of Place. New York: Alfred A. Knopf.

Kopec, Dan. 2006. Environmental Psychology for Design. New York: Fairchild Books.

Lund, Hollie. 2002. Pedestrian Environments and Sense of Community. Journal of Planning Education and Research (21), 301-312.

Lynch, Kevin. 1960. The Image of the City. Cambridge MA: MIT Press. 1976. What Time is This Place? Cambridge MA: MIT Press.. 1981. A Theory of Good City Form. Cambridge MA: MIT Press.

Mehta, Vikas. 2007. Lively Streets: Determining Environmental Characteristics to Support Social Behavior. Journal of Planning Education and Research 27(2), 143-148.

Nasar, Jack. 1998. The Evaluative Image of the City. Thousand Oaks, CA: Sage.

Norberg-Schulz, Christian. 1979. Genius Loci: Towards a Phenomenology of Architecture. New York: Rizzoli,. Rapoport, Amos. 1977. Human Aspects of Urban Form. New York: Pergamon Press. 1990. History and Precedent in Environmental Design. New York: Plenum Press.

Rossi, Aldo. 1982. The Architecture of the City. Cambridge, MA: MIT Press.

Steele, Fritz. 1981. Sense of place. Massachusetts, CBI Publishing Company, Inc.

Trowbridge, C. 1913. On Fundamental Methods of Orientation and Imaginary Maps. Science 38(990) 888-897.

Tuan, Yi-Fu. 1974. Topophilia: A Study of Environmental Perception, Attitudes, and Values. Englewood Cliffs, NJ: Prentice-Hall. 